\title{
India-based Neutrino Observatory (INO)
}

\section{Indumathi for the INO Collaboration}

The Institute of Mathematical Sciences, CIT Campus, Chennai 600 113, India

Presenter: D. Indumathi (indu@imsc.res.in)

We discuss the current status of the proposed India-based Neutrino Observatory (INO). Emphasis has been placed on the physics possibilities (both short-term and long-term) of the detector, as well as the detector R\&D status. This talk was given at the 29th International Cosmic Ray Conference, Pune, India, Aug 8th, 2005.

\section{Neutrinos: A brief overview}

We begin with a brief overview of the current status of neutrino physics.

The charge-neutral spin-1/2 neutrino was proposed by Pauli; it restores energy as well as spin-statistics conservation in nuclear beta decay. Neutrinos occur in at least three flavours, partnering the leptons $e, \mu, \tau$. The Standard Model of Particle Physics assumes neutrinos are massless, consistent with known data from beta decay. The best limits on neutrino mass today are from tritium beta decay. It is now conclusively established (from solar, atmospheric, reactor, and accelerator experiments) that neutrinos are not massless. Furthermore, neutrino flavours mix quantum-mechanically, so that, as they propagate, they exhibit the phenomenon of oscillation. This means that at least two of the masses should be distinct.

However, neutrino masses are not well-known. Oscillation studies only determine the mass-squared differences: $\Delta m_{i j}^{2}=m_{i}^{2}-m_{j}^{2}$ and the mixing angles $\theta_{i j}$. From the various experiments, various measurements/limits on these parameters exist. We have $\theta_{12} \sim 34^{\circ}, \theta_{23} \sim 45^{\circ}$, while only an upper limit exists on the across-generation mixing angle, $\theta_{13}<13^{\circ}$ (at $3 \sigma$ ). For the mass-squared differences, we have $\Delta m_{21}^{2} \sim 0.8 \times 10^{-4} \mathrm{eV}^{2}$ while $\left|\Delta m_{32}^{2}\right| \sim 2.0 \times 10^{-3} \mathrm{eV}^{2}$. This is schematically shown in Fig. 1. Astrophysical experiments also limit the total mass in all neutrino species to be $\sum_{i} m_{i}<0.7-2 \mathrm{eV}$.

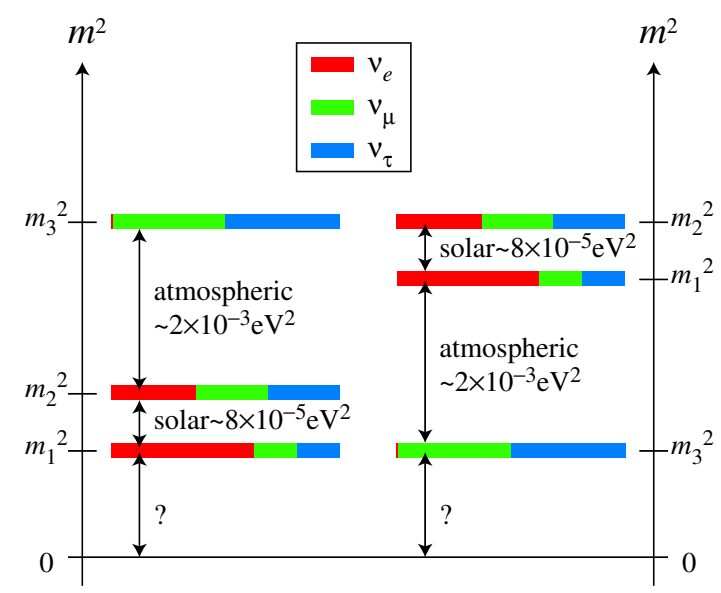

Figure 1. The schematic shows the neutrino mass-squared differences. The colour scheme denotes the extent of flavour mixing in each mass state. On the left (right) is the normal (inverted) mass hierarchy.

Most crucially, the mass ordering of the third state is not known. There are three distinct possibilities [1]: 
- $m_{1} \sim m_{2} \sim m_{3} \sim 0.2 \mathrm{eV}$ (Degenerate hierarchy)

- $m_{1}<m_{2} \ll m_{3}$ (Normal hierarchy)

- $m_{3} \ll m_{1}<m_{2}$ (Inverted hierarchy)

In short, the mass-squared differences as well as the masses are very small; the origin of small masses is a puzzle. The mass ordering (or hierarchy) is also not known. These have implications for different neutrino mass and mixing models. Furthermore, a complete oscillation pattern (with one minimum and one maximum) has not yet been directly studied in any single experiment and has only been inferred. The mixing can be large to very large, as in the (12) and (23) sectors. It is not known whether the (13) mixing is non-zero. This has a direct impact on $\mathrm{CP}$ violation in the leptonic sector.

\section{INO}

With these open issues in mind, our collaboration is studying the feasibility of locating an underground neutrino detector in India. The proposed detector is an iron calorimeter-type detector called ICAL that is capable of distinguishing the charge of particles and hence neutrino from anti-neutrino events.

The feasibility study of about 2 years duration for both the laboratory and detector is currently under-way. The program is mainly aimed at the study of atmospheric neutrinos with the possibility of acting as a future end-detector of a long-base-line experiment. Issues under study are site survey, detector R \& D, including construction of a prototype, physics studies, and human resources.

After approval is obtained, actual construction of the laboratory and ICAL detector will begin.

The possibility of using other detectors as well as addressing other physics objectives such as the nature of neutrinos (Dirac or Majorana) from experiments like neutrinoless double beta decay are also being explored. The lab should be an international facility.

\subsection{Site Selection}

Two sites were evaluated for their suitability for locating INO. They are in Singara in South India and Rammam in Northeast India.

1. The site at Singara near the town of Masinagudi (Lat 11.5 deg N, Long 76.6 deg E) under the Nilgiri Mountains in the southern peninsular shield in South India, is adjacent to a hydel project PUSHEP (Pykara Ultimate Stage HydroElectric Project). The vertical overburden is around $1.3 \mathrm{~km}$. All-around cover of more than $1 \mathrm{~km}$ exists, with the laboratory cavern to be dug at the end of a tunnel of length about $2 \mathrm{~km}$. The site is geologically stable (seismic zone 2) with uniform granitic (charnockite) rock medium of mean density 2.72 $\mathrm{gm} / \mathrm{cc}$. A detailed geological survey of the region is complete.

2. Rammam (Lat 27 deg N, Long 88 deg E) under the Himalayas, is in the Darjeeling District of West Bengal in Northeast India. A tunnel of length 3-5 km can reach an overburden of $1.4-1.8 \mathrm{~km}$. The rock quality at the tunnel/cavern location is mostly gneiss rock of mean density $2.8 \mathrm{gm} / \mathrm{cc}$ with quartz and feldspar intrusions and is in seismic zone 4. A detailed survey is now complete.

After extensive geological and geotechnical studies, and with the physics goal in mind as well as factors such as ease of access, etc., it was decided that PUSHEP is the preferred site for locating INO.

The depth at the sites is shown in Fig. 2 through the cosmic ray flux expected at the two depths.

The vertical energy-integrated flux is $2.5 \times 10^{3} / \mathrm{m}^{2} / \mathrm{sr} / \mathrm{yr}$ at PUSHEP and $1.9 \times 10^{2} / \mathrm{m}^{2} / \mathrm{sr} / \mathrm{yr}$ at Rammam. 


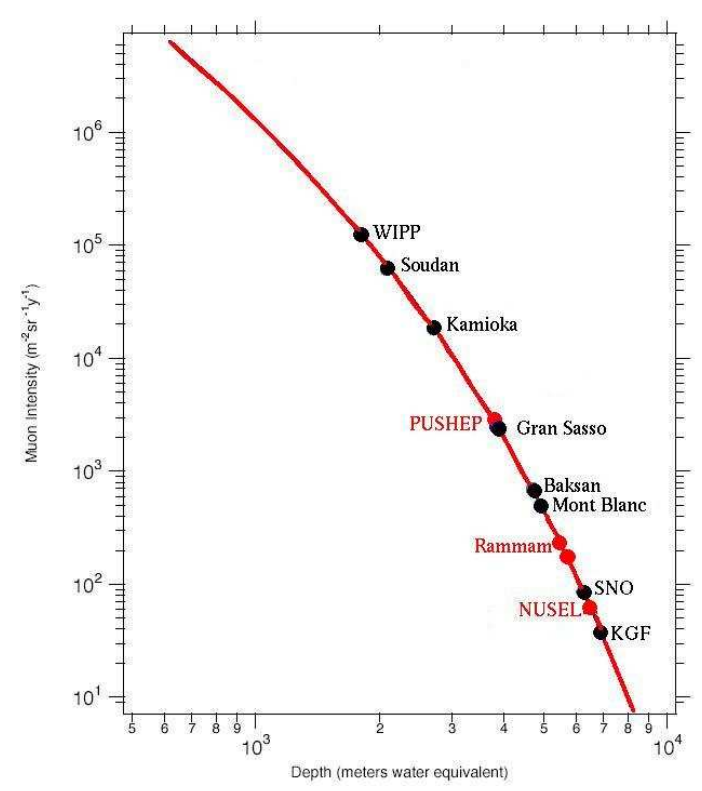

Figure 2. Muon intensity at various depths with existing underground labs and the possible locations of INO marked.

A cosmic ray background of about 3000 events/hour for ICAL at PUSHEP and roughly ten times smaller at Rammam is expected.

\subsection{The Detector}

The detector should have the following features: large target mass: 30-50 kton, (100 kton?), good tracking and energy resolution, good directionality; hence nano-second time resolution for up/down discrimination, good charge resolution, and ease of construction (modular).

The currently proposed detector is an iron calorimeter with magnetic field (ICAL); see Fig. 3 where the structural detail of mounting active detector elements is also shown. The ICAL geometry is similar to that of MONOLITH [2].
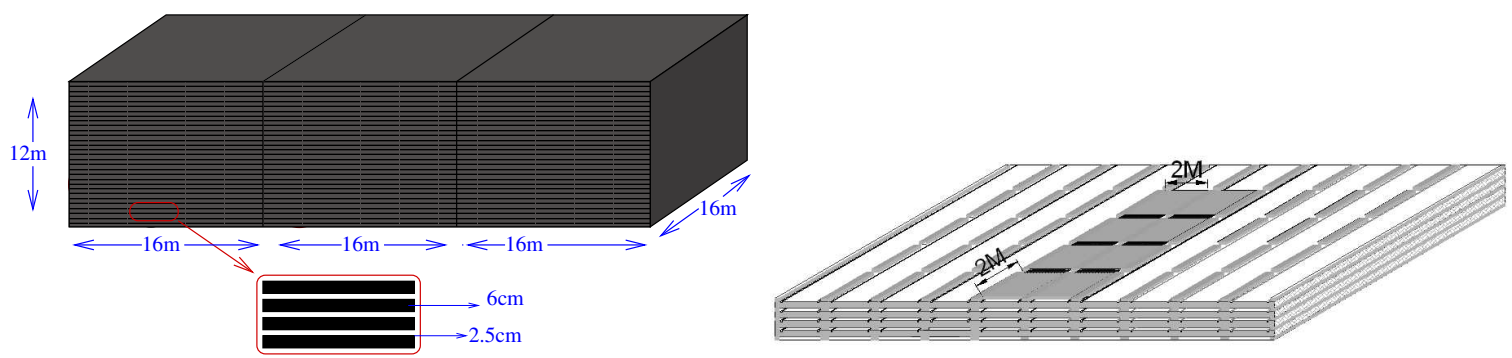

Figure 3. A schematic of the ICAL detector. On the right are shown the channels in which the RPC trays can be slid in and out.

The detector design consists of 140 layers of $6 \mathrm{~cm}$ thick iron plates, with transverse dimensions of $32 \times 16 \mathrm{~m}$, 
separated by a $2.5 \mathrm{~cm}$ air gap containing resistive plate chambers (RPCs) or glass spark chambers which are the active detector elements. Specifications are listed in Table 1. The iron is magnetised to 1-1.4 T. It will have good charge resolution and tracking and energy resolution, especially for muons. Energy of hadrons can be reconstructed as well, but rather coarsely. A larger configuration with length of $48 \mathrm{~m}$ is also being studied.

RPCs that are being tested are typically constructed with float glass, coated with graphite; see Fig. 4. A test RPC as well as a schematic for testing it using cosmic ray muons at the TIFR Institute is shown in Fig. 5.

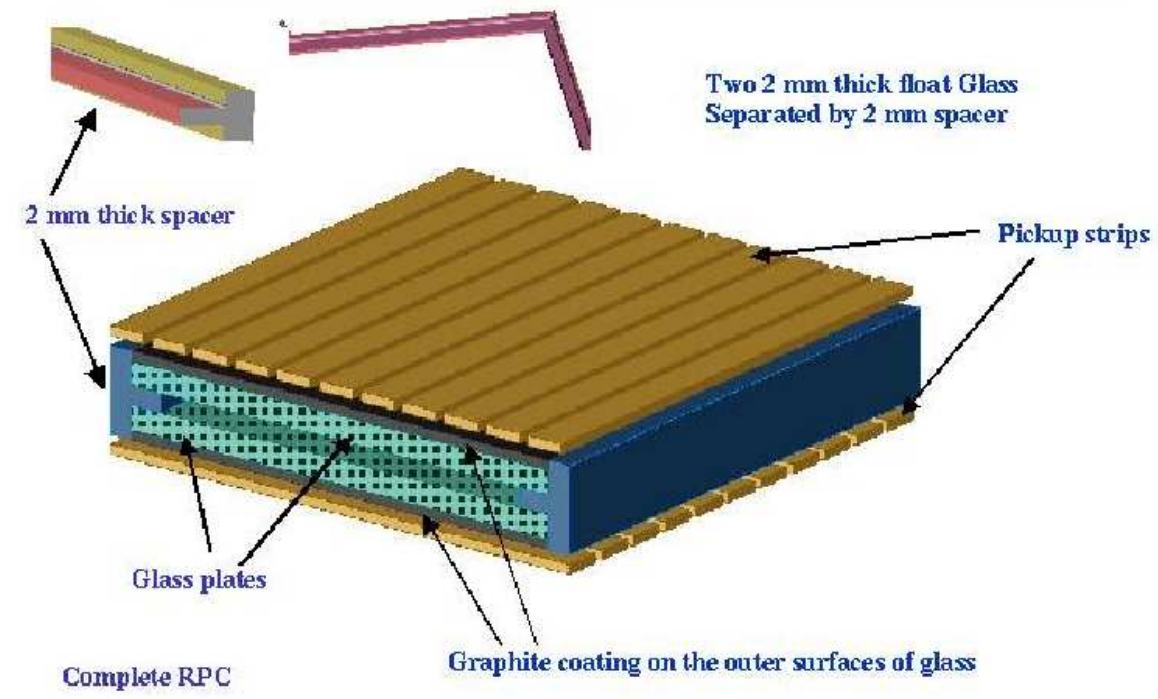

Figure 4. RPC construction with float glass and spacers. The pick-up strips (of width $2 \mathrm{~cm}$ ) are in transverse directions above and below the RPCs so that a pixel size of $2 \mathrm{~cm}$ is obtained in both $x$ - and $y$-directions.
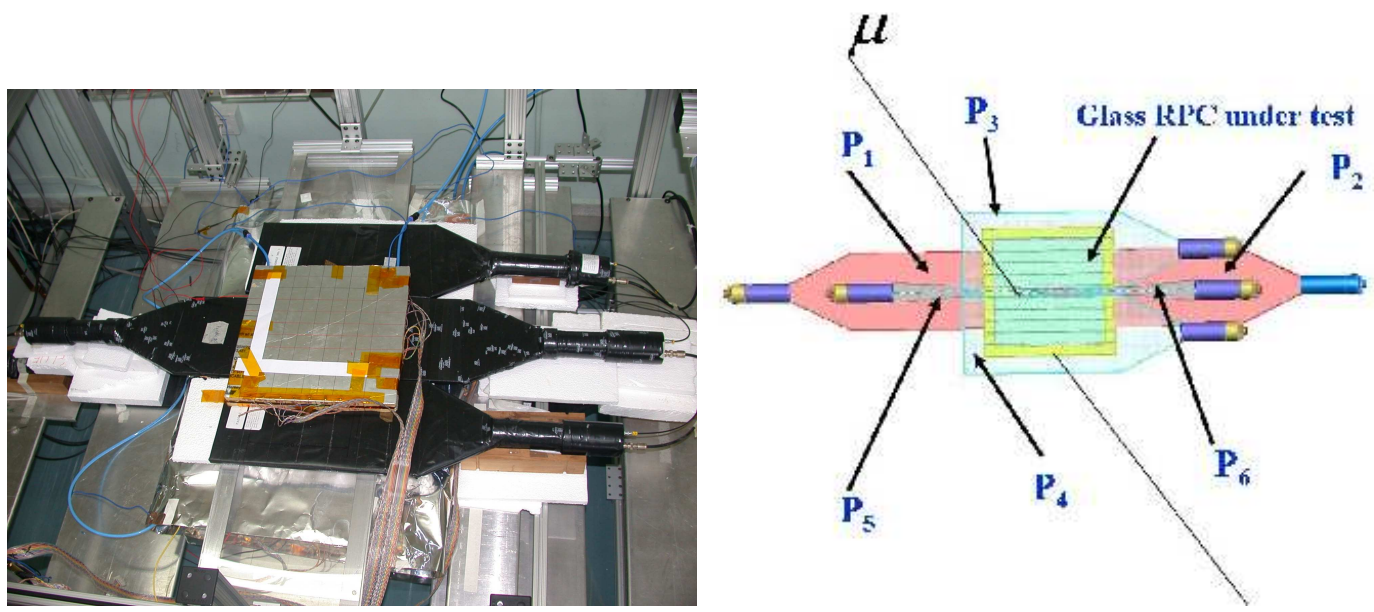

Figure 5. RPC testing facility at TIFR, Mumbai. The labels $P_{1}$ to $P_{6}$ are paddles of scintillator coupled to photomultiplier tubes, used as either triggers or vetos. 
RPC efficiency studies are being conducted with different gas mixtures (of argon, freon and isobutane (8\%)). About $90 \%$ efficiencies with nano-second timing at voltage around $9 \mathrm{KV}$ are obtained with smaller size detectors; typical results are shown in Fig. 6. Timing of around $1 \mathrm{~ns}$ have been obtained as can be seen from Fig. 6 . Larger detectors of about $1 \times 1 \mathrm{~m}^{2}$ are being tested for timing, efficiency, noise, cross-talk, etc. A major issue is the stability of the detectors and this is also being addressed.
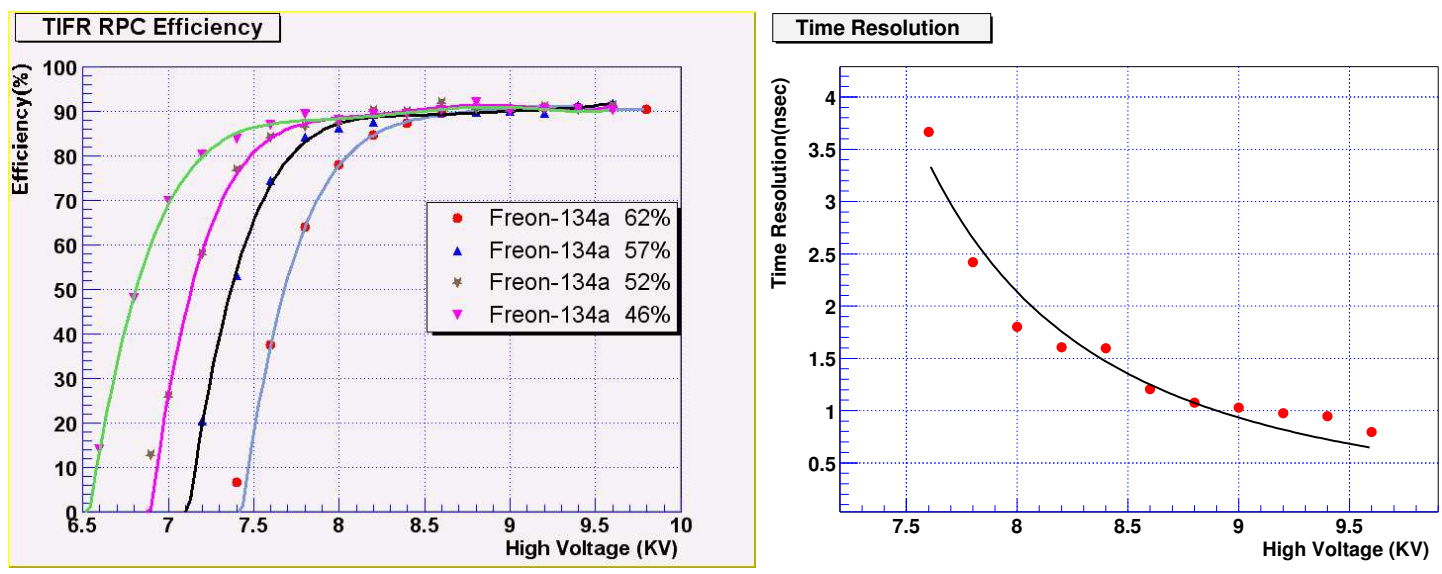

Figure 6. RPC efficiency and timing for different gas mixtures.

\begin{tabular}{|l|l|}
\hline \multicolumn{2}{|c|}{ ICAL } \\
\hline No. of modules & 3 \\
Module dimension & $16 \mathrm{~m} \times 16 \mathrm{~m} \times 12 \mathrm{~m}$ \\
Detector dimension & $48 \mathrm{~m} \times 16 \mathrm{~m} \times 12 \mathrm{~m}$ \\
No. of layers & 140 \\
Iron plate thickness & $\sim 6 \mathrm{~cm}$ \\
Gap for RPC trays & $2.5 \mathrm{~cm}$ \\
Magnetic field & 1.3 Tesla \\
\hline \multicolumn{2}{|c|}{ RPC } \\
\hline RPC unit dimension & $2 \mathrm{~m} \times 2 \mathrm{~m}$ \\
Readout strip width & $3 \mathrm{~cm}$ \\
No. of RPC units/Road/Layer & 8 \\
No. of Roads/Layer/Module & 8 \\
No. of RPC units/Layer & 192 \\
Total no. of RPC units & $\sim 27000$ \\
No. of electronic readout channels & $3.6 \times 10^{6}$ \\
\hline
\end{tabular}

Table 1. Specifications of the ICAL detector.

Magnet studies are also underway. The design criteria used are field uniformity, modularity, optimum copperto-steel ratio, and access for maintenance. A toroidal magnet design has been developed using a 3D magnet code MagNet6.0 as shown in Fig. 7. A scale model of 1:100 constructed at VECC, based on a 2D magnet code also agrees pretty well with the simulations, as shown in Fig. 8.

A prototype magnet is to be built at VECC, Kolkata. It will have 13 layers of nearly $2 \mathrm{~m} \times 2 \mathrm{~m}$ iron, about 


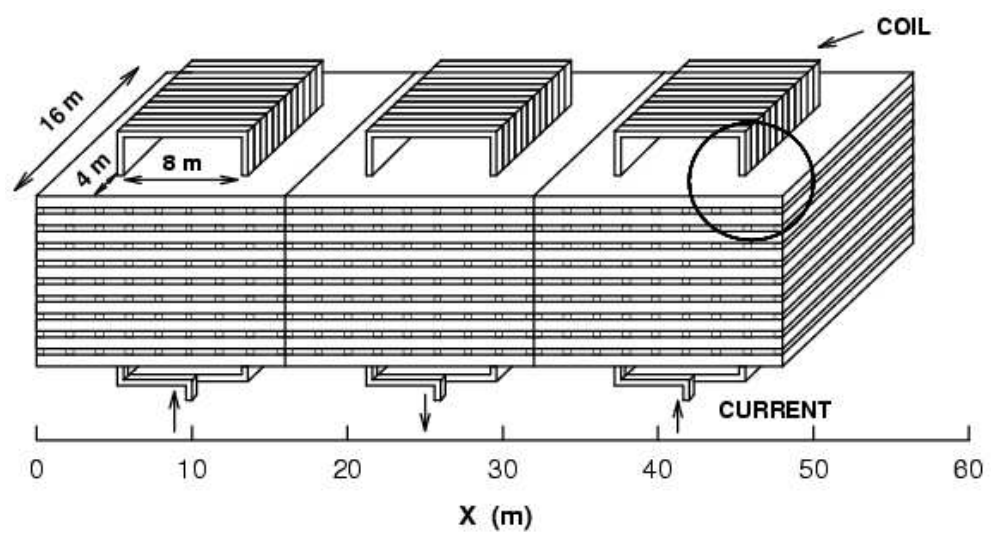

Figure 7. Proposed toroidal magnet design for ICAL.
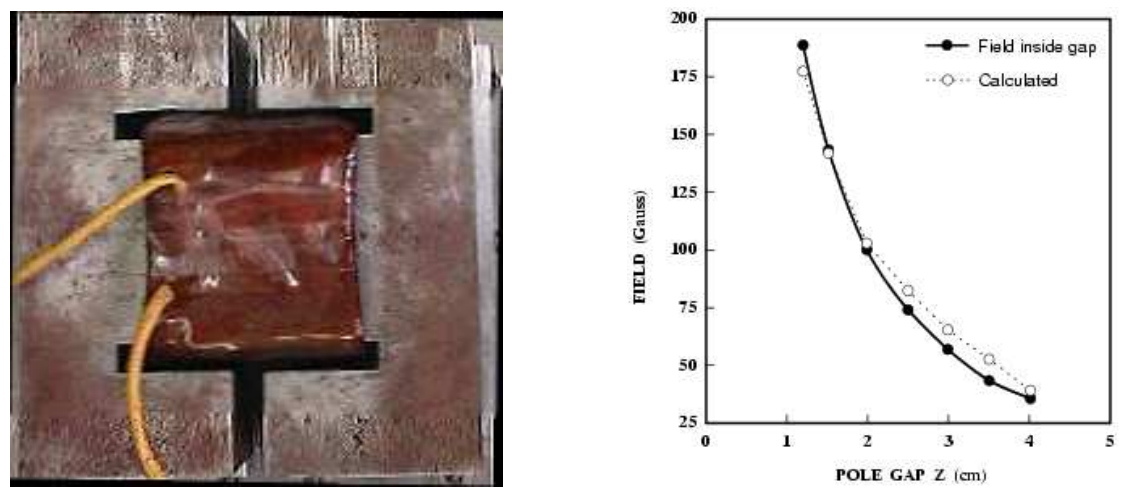

Figure 8. Results of the scaled 1:100 model and a 2D magnet code.

$5.4 \mathrm{~cm}$ thick. Since the iron that has been procured is very soft, it is hoped to be able to magnetise it up to $2 \mathrm{~T}$. However, the same coil design will be used in the prototype as for the proposed ICAL detector.

For the prototype, a gas mixing unit has been built at SINP, Kolkata as shown in Fig. 9. A schematic for the read-out electronics is also ready; see Fig. 9.

\section{Physics with Atmospheric Neutrinos}

Phase I mainly involves a study of atmospheric neutrinos with the ICAL detector. Atmospheric neutrinos, of both $e$ and $\mu$ type, have a large range in energy $E$ and path-length traversed $L$. There is an up-down symmetry in the flux of some-what higher energy neutrinos (in the absence of neutrino oscillations) so that the up-going neutrino rates in a bin around a zenith angle $\theta$ can be normalised by the down-going rates [3] in a bin around $\theta \leftrightarrow \pi-\theta$. This is demonstrated in Fig. 10 .

Events Generation : Events are generated using HONDA flux [4] with the Nuance Neutrino generator [5] with some input oscillation parameters $\Delta m_{32}^{2}, \theta_{23}$, and $\theta_{13}$. The last determines matter-dependent effects 

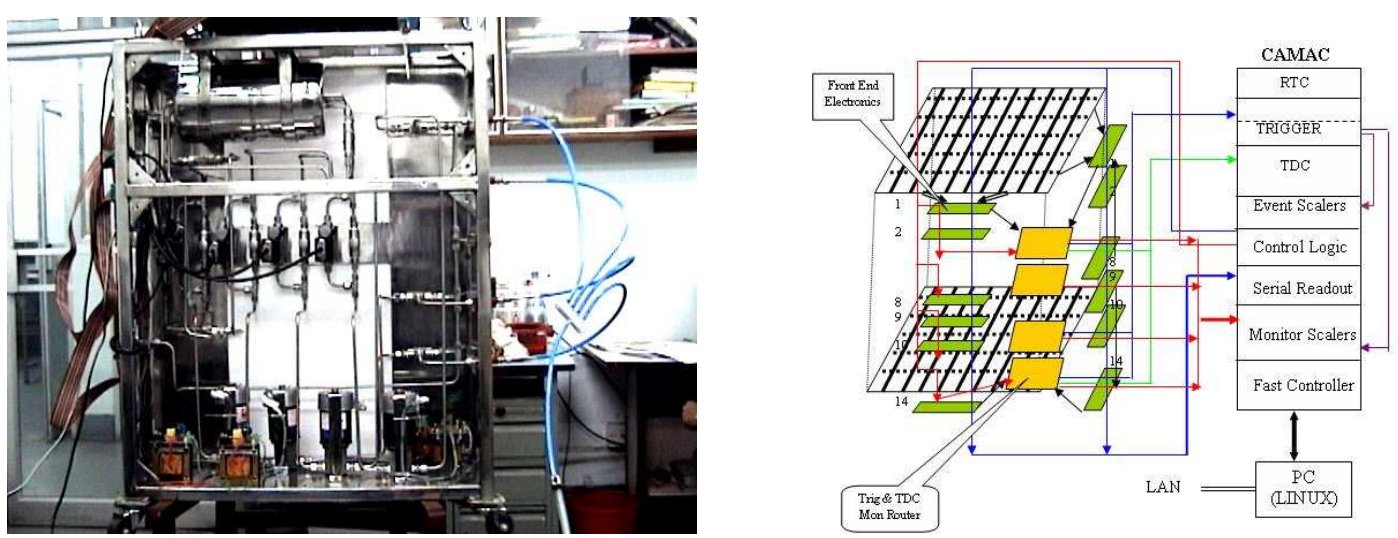

Figure 9. Left: Gas mixing unit at SINP, Kolkata. Right: A schematic of the read-out electronics for the prototype.

which can be measured from charge-identification; we will only show results with $\theta_{13}=0$ here and refer the reader to several articles that have discussed matter effects $[6,7,8]$. For neutrino energies greater than 0.8 $\mathrm{GeV}$, neutrino $\mathrm{CC}$ events of interest are generated in roughly equal proportions via quasielastic, resonant and DIS processes. All results are shown for 5 years of accumulated CC events. Typically interesting events have $E>1-2 \mathrm{GeV}$ so the proportion of DIS events in the final sample is somewhat higher than the others.

A major issue, yet to be studied, is the mis-identification of pions as muons from $\mathrm{NC}$ as well as a subset of CC events and electron CC events.

Events Analysis : In the presence of oscillations, the ratio of up-coming to down-going event rates in a given $L / E$ bin is given as

$$
\frac{\text { up rate }}{\text { down rate }}=" P_{\mu \mu} "=R \otimes\left\{1-\frac{\sin ^{2} 2 \theta_{23}}{2}\left(1-\cos 2.54 \Delta m_{32}^{2} \frac{L}{E}\right)\right\},
$$

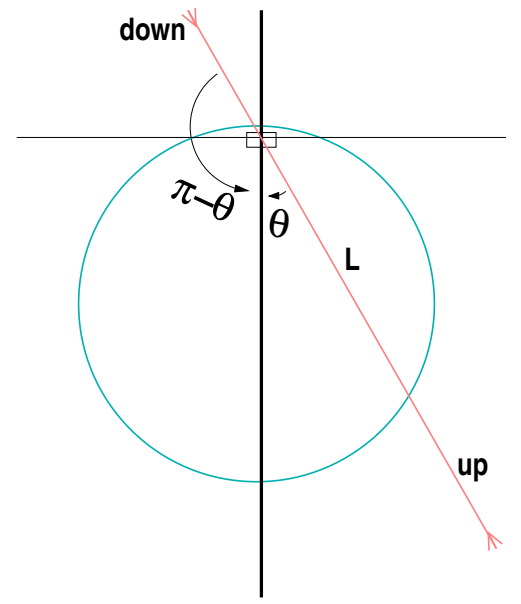

Figure 10. Mirror symmetry in zenith angle dependence of atmospheric neutrino flux implies that (at larger energies) the up-coming neutrino flux in a bin around $\theta$ is the same as that of down-going neutrinos in a bin around $(\pi-\theta)$. 
Here the up-events rate is normalised by the down-rate in a bin with a path length $\tilde{L}$ such that the corresponding zenith angle is $\theta_{\mathrm{dn}} \leftrightarrow \pi-\theta_{\mathrm{up}}$ in that bin. This ratio, which should have been close to the muon neutrino survival probability $P_{\mu \mu}$ is smeared out due to finite detector resolution effects. This smearing is particularly noticeable at large $L / E$.

The resolution function $R$ is determined by the $L / E$ resolution of the ICAL detector. So the analysis needs a knowledge of this resolution function, which depends on the quality of reconstruction of tracks in the detector. The energy of the neutrino is approximated by $E_{\nu} \sim E_{\mu}+E_{h}$ where $E_{h}$ is the total energy deposited in hadrons, while the direction of the neutrino is approximated by the muon direction alone. A typical test muon event with constant momentum is shown in Fig. 11 for a $B_{z}$ field of $1 \mathrm{~T}$. The momentum is reconstructed to within 5\% as also its direction. Hadron energy reconstruction is not as good, as can be seen in Fig. 12.

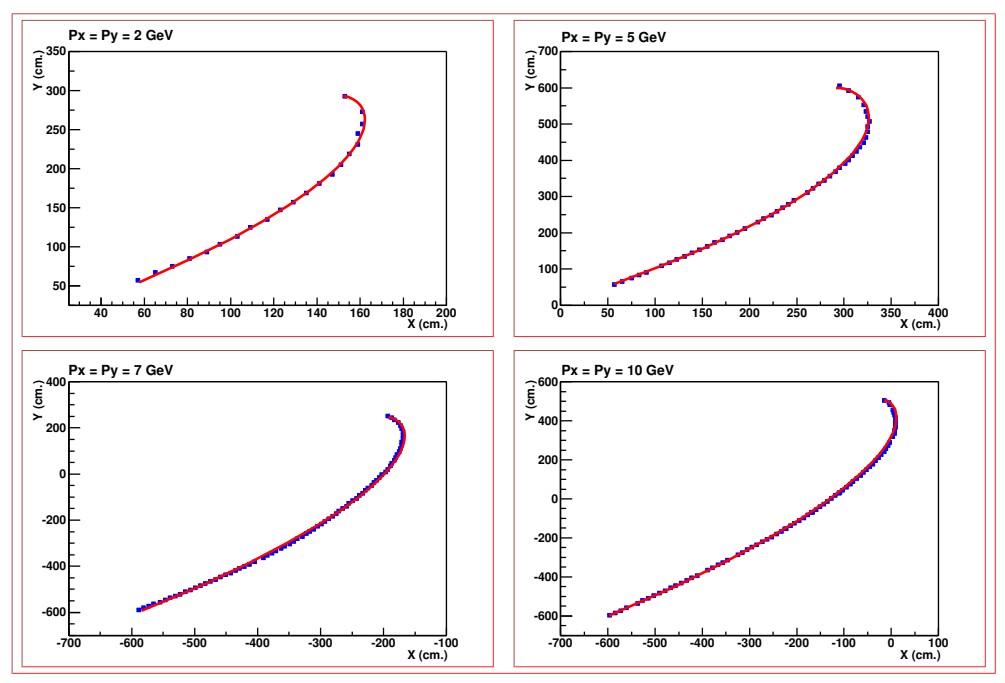

Figure 11. Tracks of hits in the transverse direction to the applied magnetic field.
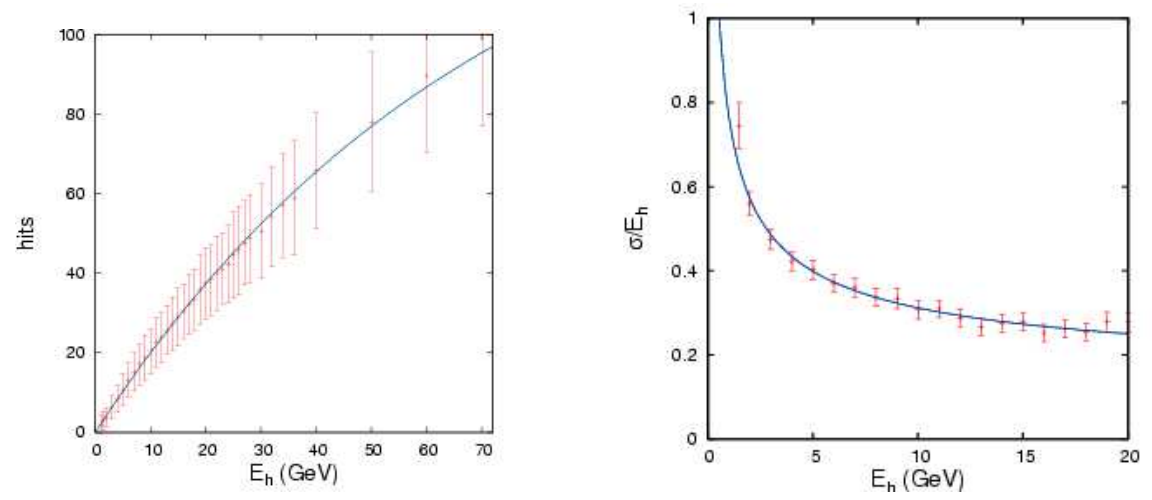

Figure 12. Number of hadron hits as a function of energy and the subsequent hadron energy resolution.

Two sets of data were analysed, with and without magnetic field. For the former, we analyse both the fully- 
contained as well as partially contained events. About $40-50 \%$ of the generated events survived the cuts (different in each case).

Results for the FC case with $B_{y}=1 \mathrm{~T}$ are shown in Fig. 13. Inputs used were $\Delta m_{32}^{2}=2 \times 10^{-3} \mathrm{eV}^{2}$; $\sin ^{2} 2 \theta_{23}=1.0$. The fits obtained were $2.02_{-0.24}^{+0.27} \times 10^{-3} \mathrm{eV}^{2} ; \sin ^{2} 2 \theta_{23}>0.96$.
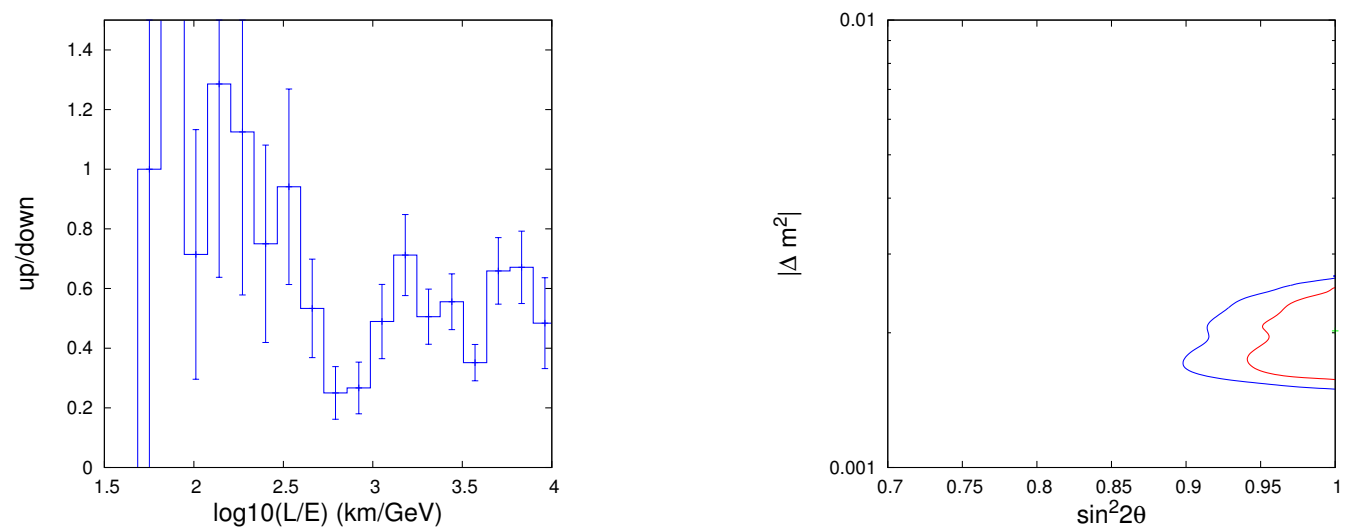

Figure 13. The up/down ratio and reconstruction of the 2-flavour neutrino allowed parameter space at 90\% and 99\% CL.

\subsection{Matter effects with atmospheric neutrinos}

Matter effects involve the participation of all three (active) flavours and hence involve both $\sin \theta_{13}$ and the CP phase $\delta$. Although sensitive to the (23) mass ordering, as seen from Fig. 14, this needs large exposures of about $800-1000$ kton-yrs.

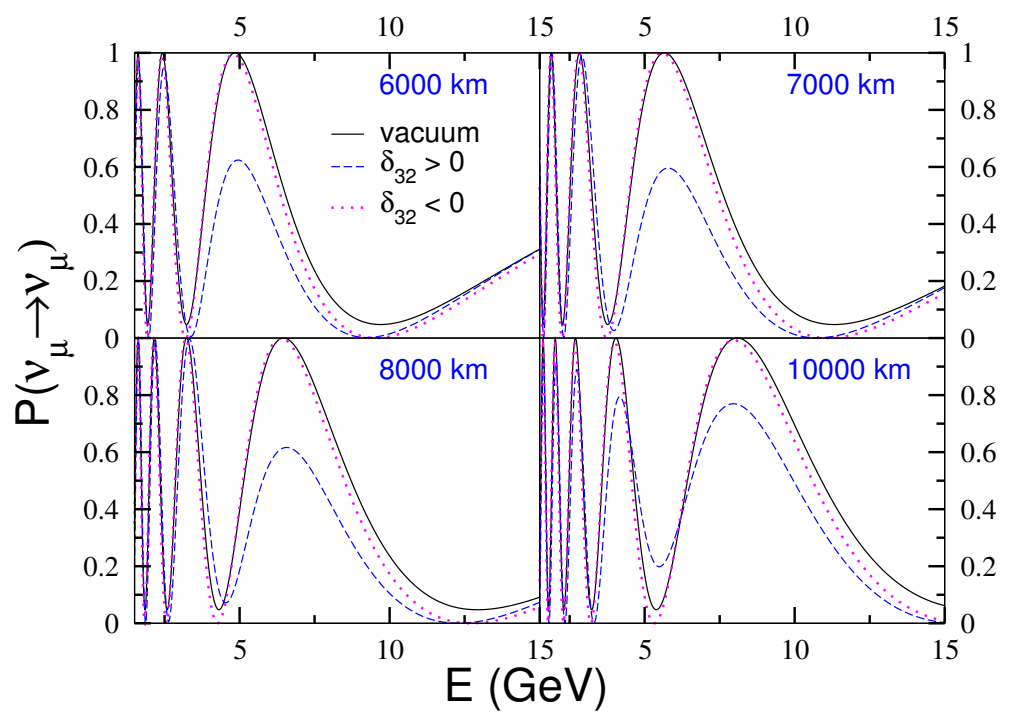

Figure 14. Matter effects distinguish the sign of $\Delta m_{32}^{2}$. 
The difference asymmetry,

$$
\mathcal{A}=\frac{U}{D}-\frac{\bar{U}}{\bar{D}}
$$

which can be thought of as a "normalised" difference of matter and anti-matter probabilities, can help enhance the matter asymmetry and hence determine the mass hierarchy, provided $\theta_{13}>6^{\circ}$, as seen in Fig. 15. Detailed studies of the mass hierarchy are in progress.

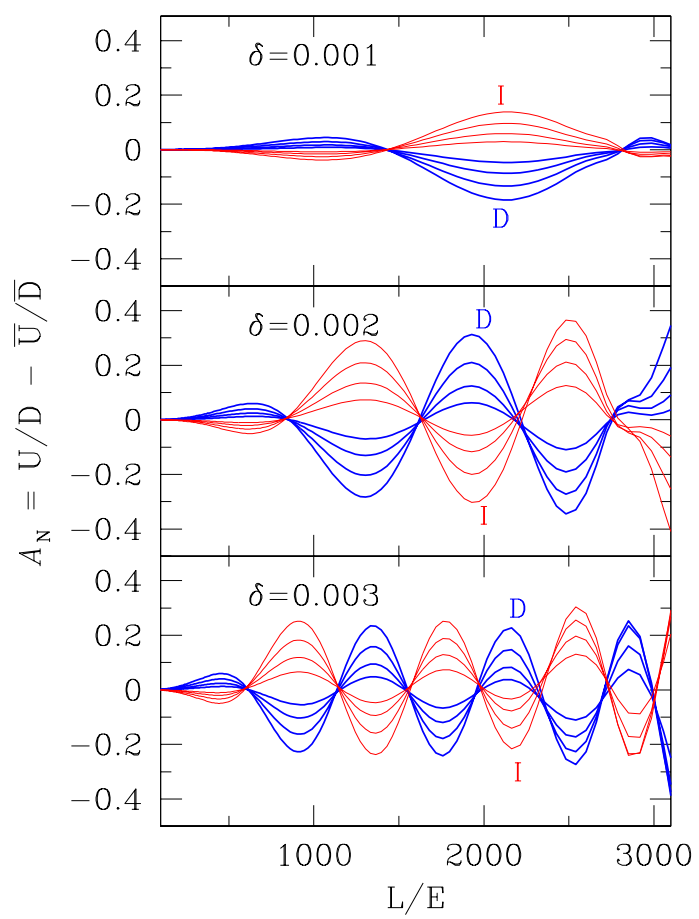

Figure 15. The difference asymmetry as a function of $L / E$ in $\mathrm{km} / \mathrm{GeV}$. The curves labelled $\mathrm{D}$ and I refer to direct and inverted hierarchies and the separation between them increases with $\theta_{13}$. Different curves correspond to $\theta_{13}=5,7,9,11^{\circ}$.

Other physics possibilities with ICAL include discrimination between oscillation of $\nu_{\mu}$ to active $\nu_{\tau}$ and sterile $\nu_{s}$ from up/down ratio in "muon-less" events in atmospheric neutrinos, probing CPT violation from rates of neutrino- to rates of anti-neutrino events in the detector, and constraining long-range leptonic forces by introducing a matter-dependent term in the oscillation probability even in the absence of $U_{e 3}$, so that neutrinos and anti-neutrinos oscillate differently.

An Interim Status Report including details of detector construction and simulation as well as site survey, was submitted to the funding authorities on May 1, 2005. 

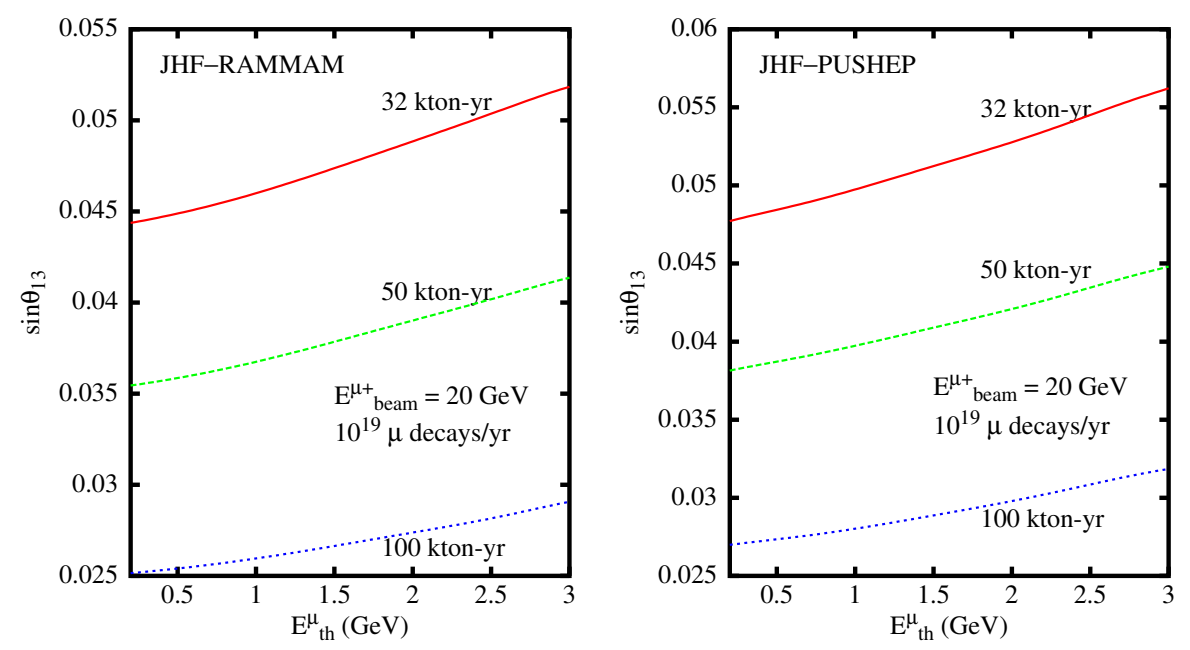

Figure 16. $\sin \theta_{13}$ reach for different muon energies for different base-lines.

\section{Stage II: Neutrino factories and INO (ICAL++)}

A burning issue in neutrino physics is whether the 1-3 mixing angle is zero or not. If $\sin \theta_{13} \neq 0$, one can look for a determination of $\sin \theta_{13}$ itself, sign of the (23) mass-squared difference $\Delta m_{32}^{2}=m_{3}^{2}-m_{2}^{2}$, CP violation through a $\mathrm{CP}$ violating phase $\delta$ that occurs in the mixing matrix when there are three active coupled neutrino species.

Such studies can be done with neutrino beams from neutrino factories (with muon storage rings). This is still far into future, but lots of work is going on (see neutrino oscillation industry web-page). INO (ICAL++) is a possible far-end detector for such long baseline experiments, particularly since charge discrimination is built in to such a detector. This is because measurements typically involve wrong sign muon detection and hence have low backgrounds. For example, suppose the beam has $\nu_{e}$ and $\bar{\nu}_{\mu}$. The $\bar{\nu}_{\mu} \rightarrow \mu^{+}$in the detector and is directly detected. During propagation, oscillations can cause a conversion of beam $\nu_{e} \rightarrow \nu_{\mu}$. Such neutrinos are detected from $\nu_{\mu}$ (osc-beam) $\rightarrow \mu^{-}$in the (detector). 10 such wrong-sign events/kton is considered a signal for oscillations.

Note: Since ICAL is not very sensitive to electrons, the mode in which the wrong-sign event is from electron detection (sensitive to $P_{\mu e}$ ) is not considered here.

For such studies a muon detection threshold of $2 \mathrm{GeV}$ and a muon energy resolution of $5 \%$ was assumed. The figures show the reach of various base-lines with ICAL (or an upgraded ICAL) as the far-end detector. Fig 16 shows the reach for $\theta_{13}$ for different base-lines from JHF to Rammam and to PUSHEP.

Fig. 17 shows the number of wrong-sign muons as a function of $\Delta m_{32}^{2}$ that enables determination of the (23) mass ordering for a neutrino factory located at JHF, with detectors at Beijing, PUSHEP and Rammam.

Fig. 17 also shows the variation of the ratio of $\mu^{+} / \mu^{-}$rates with the CP phase $\delta$ for JHF to Rammam and PUSHEP and FermiLab to Rammam and PUSHEP. It is seen that the JHF-PUSHEP baseline is near magic, that is, close to the baseline of about $7200 \mathrm{~km}$ where the oscillation probabilities are unaffected by the $\mathrm{CP}$ phase. All matter-antimatter asymmetry for this base-line arises purely from matter effects. Combining this with another base-line where $\mathrm{CP}$ effects are non-vanishing can provide a clean separation of matter and $\mathrm{CP}$ violation effects. 

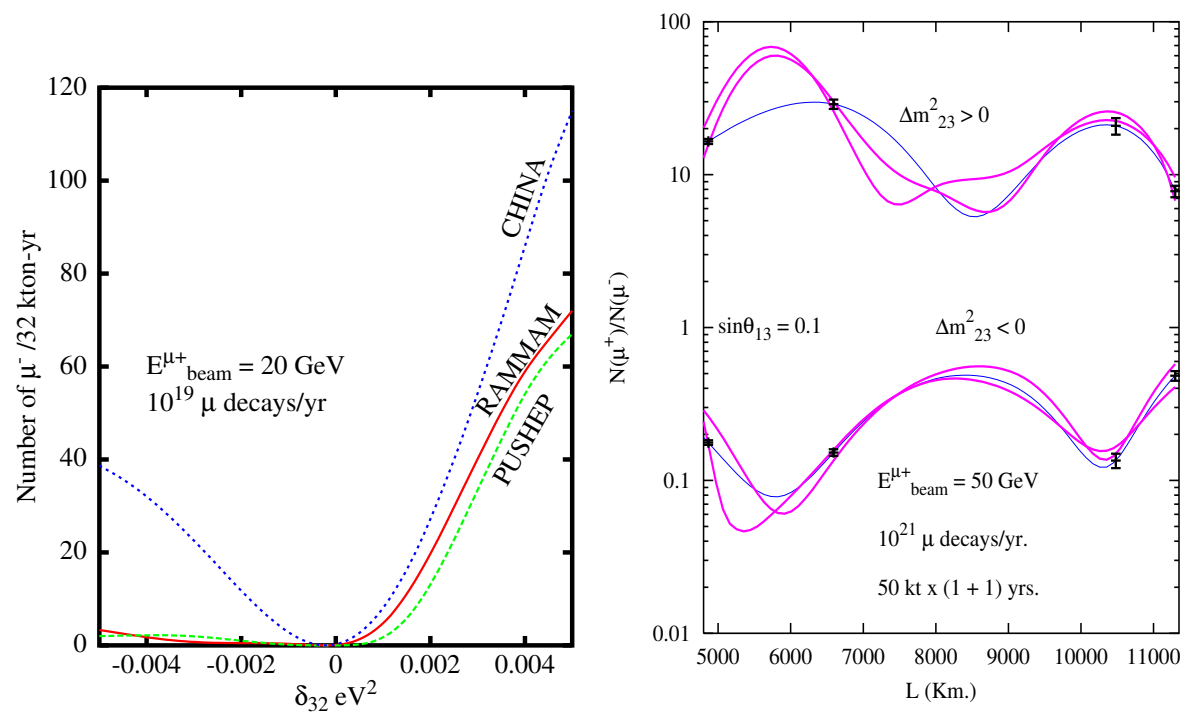

Figure 17. Left: Reach for determining the (23) mass ordering. Right: Dependence on CP phase $\delta$.

Other studies at INO include a preliminary study of neutrino-less double beta decay. A working group is looking at the possibility of cryogenic detection to measure DBD in ${ }^{124} \mathrm{Sn}$ and ${ }^{150} \mathrm{Nd}$.

\section{Outlook}

The proof-of-principle working of RPC has been shown. Magnet studies are under-way. Construction of a prototype is the immediate goal. Two possible sites exist; both seem good options. PUSHEP in South India has been decided on as the preferred site. Simulations of atmospheric neutrinos are being done.

A germane atmospheric neutrino programme can be conducted with ICAL as it is sensitive to oscillation parameters to better accuracy than current Super-K [9]. Also, it may have the edge on MINOS [10, 11] if $\Delta m_{32}^{2}$ is smaller than expected. ICAL will be sensitive to matter effects and the 2-3 mass ordering if $\sin ^{2} 2 \theta_{13}>0.05$. Hence it can settle the mass hierarchy. It can also participate in a substantial neutrino factory programme, in the future.

In short, the outlook looks good! This is a massive project: we welcome active collaboration both from within India and abroad.

\section{Acknowledgements}

I thank the organisers of the 29th ICRC conference for giving me the opportunity to present this talk.

\section{References}

[1] APS multi-divisional neutrino study, physics/0411216, 2004. 
[2] N.Y. Agafonova et al., The MONOLITH proposal, LNGS P26/2000, CERN/SPSC 2000-031, August 2000 (http://castore.mib.infn.it/ monolith/proposal/).

[3] P. Picchi and F. Pietropaolo, ICGF RAP. INT. 344/1997, Torino 1997 (CERN preprint SCAN-9710037).

[4] M. Honda, T. Kajita, K. Kasahara, S. Midorikawa, Phys. Rev. D 64053011 (2001).

[5] The NUANCE Neutrino Generator, D. Casper, Nucl. Phys. Proc. Suppl. 112161 (2002) (http://www.ps.uci.edu/ nuint/nuance/default.htm).

[6] Sergio Palomares-Ruiz and S. T. Petcov, arXiv: hep-ph/0406096

[7] D. Indumathi and M.V.N. Murthy, arXiv: hep-ph/0407336

[8] Raj Gandhi, Pomita Ghoshal, Srubabati Goswami, Poonam Mehta, S Uma Sankar, arXiv: hep$\mathrm{ph} / 0408361$

[9] Masaki Ishitsuka, for the Super-K Collab., talk given in NOON04, Feb 2004

[10] A. Para, arXiv: hep-ph/0005012; C. Andreopoulos, P. Stamoulis and G. Tzanakos, NuMI-Note-ATM_NU990, UA/ Phys/ HEP/ 11-05-2003 (http://www-numi.fnal.gov/).

[11] Y. Itow et al., arXiv: hep-ex/0106019 (http://neutrino.kek.jp/jhfnu/). 
